\title{
Artículo
}

\section{Estrategias de las universidades españolas para mejorar el rendimiento en matemáticas del alumnado de nuevo ingreso}

\author{
Luis J. Rodríguez-Muñiz* y Patricia Díaz \\ Departamento de Estadística e I.O. y Didáctica de la Matemática, Facultad de Ciencias, Universidad de Oviedo, Oviedo, España
}

\section{INFORMACIÓN DEL ARTÍCULO}

\section{Historia del artículo:}

Recibido el 31 de octubre de 2014

Aceptado el 12 de enero de 2015

On-line el 24 de febrero de 2015

\section{Palabras clave:}

Aprendizaje on-line

Colaboración docente

Curso cero

Matemáticas

Universidad

\begin{abstract}
R E S U M E N
En los últimos años las universidades españolas han desarrollado diferentes actividades para mejorar el rendimiento académico del alumnado de nuevo ingreso en asignaturas de matemáticas en los primeros cursos. El objetivo de este artículo es realizar un análisis sobre las características de estas actuaciones, con el fin de valorar su eficiencia. Para ello, mediante entrevistas on-line, presenciales y telefónicas, se han recabado datos de 34 universidades públicas con metodología presencial, sobre actividades desarrolladas principalmente durante el primer curso universitario, en aquellas titulaciones con asignaturas de matemáticas en sus planes de estudio. Las actividades estudiadas han sido: cursos cero, de acción tutorial específicos de matemáticas, formación de profesorado universitario sobre bachillerato, plataformas web de aprendizaje de matemáticas, colaboración con sociedades de profesores, grupos de trabajo mixtos y actividades para colectivos específicos (estudiantes procedentes de Formación Profesional o mayores de 25/40/45 años). La información obtenida es muy variada, aunque debe reseñarse una enorme parcelación de la información y una escasa programación por competencias de las actividades. Como producto final, el artículo recoge un catálogo de buenas prácticas, así como unas propuestas de mejora de algunos aspectos detectados durante el estudio.

(C) 2014 Instituto de Ciencias de la Educación de la Universidad de Oviedo. Publicado por Elsevier España, S.L.U. Este es un artículo Open Access bajo la licencia CC BY-NC-ND (http://creativecommons.org/licenses/by-nc-nd/4.0/).

\section{Strategies in Spanish universities to improve incoming students' mathematical performance}

\begin{abstract}
A B S T R A C T
Spanish universities, over the past few years, have developed different kinds of activities in order to reduce failure rates of incoming students in mathematical courses in first year of undergraduate studies. The aim of this work is to perform an analysis of these activities, focusing on efficiency. For this purpose, by means of several email, telephone and face-to-face interviews, data from 34 public universities, with in-person teaching, have been collected regarding activities mainly developed during the first year of undergraduate studies having mathematical courses in their syllabi. The activities studied were, warm up/foundation courses, counseling plans specifically oriented for mathematics, training about secondary education training for university teachers, teaching-learning online platforms, collaboration with teacher societies, mixed working groups, and activities for special students (people older than 25/40/45 years old, and students from vocational training). The information obtained varies widely, but it is important to underline the wide fragmentation of information, and the lack of competence-oriented programs. Finally, the article presents a list of good practices, as well as several proposals for improving the different details detected within the study.
\end{abstract}

(C) 2014 Instituto de Ciencias de la Educación de la Universidad de Oviedo. Published by Elsevier España, S.L.U. This is an open access article under the CC BY-NC-ND license (http://creativecommons.org/licenses/by-nc-nd/4.0/).

\footnotetext{
* Autor para correspondencia. Facultad de Ciencias. c/ Calvo Sotelo s/n, 33007 Oviedo (España). Tel.: +34985181902; fax: +34985103353. Correo electrónico: luisj@uniovi.es (L.J. Rodríguez-Muñiz).
} 
El paso desde el bachillerato a la universidad produce un salto cualitativo de vital importancia en el alumnado. No solo cambia el tipo de atención recibida, pasando a ratios de estudiantes/profesor que pueden llegar a cuadruplicar las de bachillerato en los grandes grupos de clases expositivas, sino que también cambian los métodos docentes y las estrategias de aprendizaje. Por ello, esta transición ha sido estudiada cada vez con más detenimiento, incluso con la creación de revistas científicas especializadas en este tránsito (International Journal of First Year in Higher Education, por ejemplo).

Existe literatura dedicada a estudiar cómo las políticas de orientación y apoyo tutorial mejoran el rendimiento de los nuevos universitarios (cf. Ayuga et al., 2008; Barrenetxea et al., 2008; Taylor, 2008; Vieira y Vidal, 2006). Igualmente, se ha señalado la importancia de la acción tutorial, entendida de un sentido amplio y en el ámbito concreto de la tutoría entre iguales o mentoría (cf. Sánchez et al., 2008; Velasco y Benito, 2011).

Ciñéndose al campo de las matemáticas, se ha señalado con frecuencia el importante salto que el estudiantado percibe al cambiar desde el bachillerato (o etapa equivalente) a la universidad (véase IV Escuela de Educación Matemática Miguel de Guzmán, 2008). De Guzmán, Hogdson, Robert y Villani (1998, p. 747) indican que: "...transition often presents major difficulties for an important part of those students who take mathematics courses at the tertiary level.» Se trata de una percepción generalizada entre el profesorado y asumida por las instituciones, a pesar de la preeminencia que las matemáticas tienen en el Bachillerato (Artigue, 2001).

Diversos autores han subrayado la importancia del problema de adaptación de los nuevos estudiantes al contexto matemático en la Universidad (Barnard, 2003; McInnes, James y Hartley, 2000; Wood, 2001), centrándose algunos estudios en las dificultades concretas de un tema, si bien la mayoría de los trabajos se focalizan en la preparación previa del alumnado y su impacto en los resultados que obtiene en la Universidad (Anthony, 2000; Hourigan y O'Donoghue, 2007; Kajander y Lovric, 2005; Selden, 2005).

Clark y Lovric (2008, p.31) estudian la transición secundariauniversidad siguiendo el modelo de los ritos de paso y destacan la necesidad de tiempo para asimilar los cambios como una de las claves del éxito del proceso: "First-year university can be viewed as the incorporation phase of a rite of passage. [...] any significant change requires time to be fully accepted and built into one's cognitive, social, psychological (and other) frameworks".

En el marco educativo español se halla la literatura ya referida sobre actuaciones concretas desarrolladas en determinadas universidades, pero no existe un estudio comparativo en el ámbito de las matemáticas que atienda a las peculiaridades de esta disciplina. Los autores lideraron un proyecto de estudios y análisis del Ministerio de Educación, denominado "Análisis y valoración de las actuaciones de las universidades españolas para mejorar las competencias matemáticas de los estudiantes de nuevo ingreso», en el cual se examinaron las actuaciones realizadas en las universidades españolas para mejorar el rendimiento académico, o reducir el fracaso, de los estudiantes de nuevo ingreso en matemáticas. Algunos resultados preliminares fueron recogidos en Díaz y Rodríguez-Muñiz (2013). En el presente artículo se condensan los resultados principales del proyecto: un estudio descriptivo de la situación actual respecto a lo que se está haciendo en la Universidad española, combinado con un estudio analítico con el fin de identificar buenas prácticas. Se estudian actuaciones previas al ingreso en la Universidad pero también aquellas sostenidas durante el primer año universitario, basándose en la propuesta de modelo de transición de Clark y Lovric (2008).

El objetivo principal de este trabajo es la descripción y el análisis de las actividades dirigidas a la transición en matemáticas entre el bachillerato y la universidad, enfocado en 4 aspectos básicos: su efectividad, el tipo de trabajo por competencias desarrollado, la evaluación de las actividades y del personal implicado en ellas y, finalmente, la financiación que reciben y su coste.

Como resultado de la investigación, se ha elaborado un catálogo de buenas prácticas, tanto de aquellas que ya se están realizando en algunas universidades españolas, como otras propuestas de actuación que conlleven un trabajo proactivo en el refuerzo del estudio de las matemáticas por parte de los estudiantes de nuevo ingreso.

\section{Método}

\section{Participantes}

La población está compuesta por las universidades españolas, tanto públicas como privadas y de la Iglesia católica, constituidas y que imparten docencia en el momento de realizar el estudio (enero-septiembre de 2012). De entre las públicas se excluyeron la Universidad Nacional de Educación a Distancia (UNED) por su metodología no presencial, y la Universidad Internacional Menéndez y Pelayo y la Internacional de Andalucía por no impartir estudios de grado. Se contó, por lo tanto, con 46 universidades públicas. De entre las universidades privadas se excluyó a las que únicamente ofrecen formación on-line (Universidad a Distancia de Madrid, Universidad Internacional de La Rioja, Universitat Oberta de Catalunya y Valencia International University), las restantes fueron 22. En total, suman 68 universidades entre públicas y privadas.

Se planteó un estudio censal, enviando la petición de datos al conjunto de la población. Respondió el 74\% de las universidades públicas (es decir, 34 universidades). En el caso de las universidades privadas, la participación ha sido mucho menor, solo 3 de las 22 universidades privadas españolas han proporcionado datos al estudio.

\section{Instrumentos de evaluación}

Para recopilar la información se celebraron entrevistas semiestructuradas y personalizadas, bien con encuentros presenciales en la universidad correspondiente, bien mediante entrevista telefónica o correo electrónico.

El abanico de actuaciones que realizan las universidades españolas con el fin de mejorar el rendimiento académico relacionado con la competencia matemática de su alumnado es muy amplio, por lo que se ha agrupado del siguiente modo con el fin de optimizar el análisis: cursos cero de matemáticas, plataformas web de aprendizaje, planes de acción tutorial específicamente relacionados con matemáticas, colaboración con sociedades de profesores de matemáticas, grupos de trabajo o cursos paralelos a la docencia reglada, formación específica para profesorado universitario sobre educación secundaria, actividades dirigidas a colectivos específicos.

Conviene precisar algunos de los términos para acotar el objeto de estudio. Los cursos cero surgen en los años 90, al evidenciarse el salto entre los anteriores planes de estudio universitarios y el nuevo bachillerato surgido de la Ley Orgánica General del Sistema Educativo (LOGSE), que había concentrado (o suprimido) algunos de los anteriores contenidos del Bachillerato Unificado Polivalente (BUP) y Curso de Orientación Universitaria (COU). Así concebidos, suelen ser optativos para el alumnado y se celebran en los días previos al arranque del curso lectivo oficial, de un modo intensivo. Sin embargo este modelo intensivo, optativo y previo comienza a presentar variantes como cursos virtuales o en paralelo a la docencia y cursos transformados en asignaturas del plan de estudios.

Las plataformas web de aprendizaje son herramientas on-line que facilitan conceptos, procedimientos y actitudes que permitan al alumnado salvar las dificultades matemáticas que se encuentran en su primer año universitario. No deben confundirse con los llamados campus virtuales, que suelen estar vinculados al 
material docente de una asignatura concreta. Las plataformas referidas tienen un carácter más transversal, no suelen estar dedicadas a una asignatura específica y suelen abordar contenidos directamente relacionados con el Bachillerato y no con la universidad. Las plataformas web suelen incorporar contenidos, demostraciones animadas y cuestionarios que permiten al alumnado la realización de autoevaluaciones para conocer su grado de aprendizaje.

Los planes de acción tutorial se desarrollan en las universidades españolas desde mediados de los años 90, y suelen ser holísticos en su concepción del proceso de enseñanza-aprendizaje, si bien algunos de ellos se centran especial o únicamente en los problemas surgidos en el aprendizaje de las matemáticas. Ellos son el objeto de estudio en el caso de este artículo.

Las sociedades de profesores de matemáticas agrupan en los ámbitos regional y nacional al profesorado de esta disciplina y realizan numerosas actividades en el ámbito del desarrollo profesional del profesor o la divulgación científica; a ellas pertenece profesorado de secundaria y de universidad.

Por grupos de trabajo se entienden todas aquellas agrupaciones de profesorado o profesorado y alumnado que trabajan colaborativamente de forma estable en alguna actividad matemática fuera del ámbito de la docencia reglada.

La formación del profesorado universitario suele articularse a través de los Institutos de Ciencias de la Educación u organismos similares en el seno de cada universidad. Habitualmente desarrollan cursos tanto generales como específicos. El objeto de estudio en este artículo son las actividades formativas específicas sobre la formación inicial del futuro alumnado universitario, concretamente en el ámbito matemático: qué matemáticas se estudian y cómo se estudian en la Educación Secundaria Obligatoria y postobligatoria.

Por último, desde la reforma del acceso a la universidad implantada en 2010, aparecen distintos perfiles de alumnado, algunos de ellos con unas características muy específicas respecto a su formación inicial. Entre ellos se encuentra el alumnado procedente de ciclos formativos de grado superior, el alumnado que supera las pruebas de acceso para mayores de 25 años, mayores de 40 con experiencia profesional o mayores de 45 (que no puede tener otra titulación habilitante para el acceso a la universidad) o el alumnado procedente de sistemas educativos extranjeros.

Para homogeneizar los datos recogidos, se diseñaron unas fichas de evidencias para cada una de las actividades anteriores, identificando los principales ítems de interés para el estudio. La tabla 1 muestra los principales ámbitos de interés de cada actividad.

\section{Procedimiento}

La recogida de información se realizó utilizando como base las fichas de evidencias. Los interlocutores para proporcionar la información requerida en los cuestionarios fueron los responsables de cada una de las actividades en cada universidad. En algunos casos una sola persona o servicio coordinaba todas las actuaciones (suele tratarse de un vicerrectorado o del departamento de matemáticas). En otros casos, la mayoría, cada actividad era coordinada por una persona o servicio: una facultad (a través del decanato), un departamento (generalmente el de matemáticas), un servicio transversal de orientación académica, o simplemente profesorado encargado de dicha actividad a título individual.

Las actividades sobre las que se solicitó información fueron las realizadas en el curso 2011-2012 y las ya realizadas o proyectadas para el curso 2012-2013. La baja participación de las universidades privadas (solo 3 respuestas) ha motivado que no se hayan tenido en cuenta a la hora de realizar el análisis, dada además su autonomía en los procesos de admisión del alumnado. Por ello, el estudio se ha ceñido a las universidades públicas con metodología presencial en estudios de grado.
Tabla 1

Ítems de interés de las actividades estudiadas

\begin{tabular}{|c|c|}
\hline \multicolumn{2}{|l|}{ Cursos cero } \\
\hline Información al alumnado & Programación por competencias \\
\hline Tasas de matrícula & Docentes del curso \\
\hline Financiación & Método de selección de los docentes \\
\hline Duración & Formación recibida por los docentes \\
\hline Ubicación en el calendario & Profesorado universitario o de \\
\hline Organizadores & secundaria \\
\hline Materia que se imparte & Evaluación del curso \\
\hline \multicolumn{2}{|l|}{$\begin{array}{l}\text { Enfoque a un grado concreto } \\
\text { o generalistas }\end{array}$} \\
\hline \multicolumn{2}{|l|}{ Plataformas web de aprendizaje } \\
\hline Existencia de la plataforma & Respuesta del alumnado \\
\hline $\begin{array}{l}\text { Quién la crea, mantiene y } \\
\text { actualiza }\end{array}$ & $\begin{array}{l}\text { Condiciones de uso: libre o bajo } \\
\text { usuario }\end{array}$ \\
\hline Destinatarios & Programación por competencias \\
\hline Tiempo en funcionamiento & Evaluación de la plataforma \\
\hline Existencia de estudios de uso & \\
\hline \multicolumn{2}{|c|}{ Planes de acción tutorial (PAT) directamente relacionados con matemáticas } \\
\hline Existencia de un PAT & Periodicidad de reuniones \\
\hline Organizadores & Existencia de reconocimiento al tutor \\
\hline Actividad obligatoria o & Formación de los tutores \\
\hline voluntaria & Tipo de financiación \\
\hline Tutores y tutorados & Programación por competencias \\
\hline Número de alumnos/tutor & Estudios sobre el rendimiento \\
\hline Duración & académico \\
\hline Información suministrada & Evaluación \\
\hline \multicolumn{2}{|l|}{ Grupos de trabajo } \\
\hline Organizadores & Presencialidad \\
\hline Docentes involucrados & Necesidad de matrícula \\
\hline Número de grupos de trabajo & Convalidación de créditos \\
\hline Ratio alumnos/grupo & Tipo de financiación \\
\hline Destinatarios & Coste \\
\hline Tipo de actividades & Programación por competencias \\
\hline Distribución temporal & Evaluación de los grupos de trabajo \\
\hline \multicolumn{2}{|c|}{ Formación del profesorado universitario de matemáticas } \\
\hline Formación recibida por los & Existencia de publicaciones conjuntas \\
\hline docentes universitarios sobre & Realización de foros, reuniones o \\
\hline $\begin{array}{l}\text { secundand. lipo y } \\
\text { periodicidad }\end{array}$ & Trabajo por competencias \\
\hline Participación de profesorado & Estudios sobre rendimiento académico \\
\hline universitario en formación & Mejora de conocimiento mutuo \\
\hline de profesorado de secundaria & Evaluación \\
\hline $\begin{array}{l}\text { Reconocimiento de la } \\
\text { formación }\end{array}$ & Tipo de financiación \\
\hline $\begin{array}{l}\text { Grupos de trabajo estables } \\
\text { entre secundaria y } \\
\text { universidad }\end{array}$ & \\
\hline \multicolumn{2}{|c|}{ Colaboración con sociedades de profesores de matemáticas } \\
\hline Existencia de colaboración & Tipo de colaboración y actividades \\
\hline Sociedades colaboradoras & organizadas \\
\hline \multicolumn{2}{|c|}{ Actividades dirigidas a colectivos específicos } \\
\hline Organizadores & Tipo de financiación \\
\hline Docentes implicados & Coste \\
\hline Actividades realizadas & Presencialidad \\
\hline Perfil del alumnado & Programación por competencias \\
\hline Necesidad de matrícula & Evaluación \\
\hline
\end{tabular}

Fuente: Elaboración propia.

Análisis de los datos

A tenor del tipo de datos recogidos, el análisis que se ha realizado es descriptivo, en términos de porcentajes, identificando el tipo de actuaciones más frecuentes y las características más comunes a cada una de ellas.

\section{Resultados}

Con las limitaciones al alcance que se señalarán en la discusión, la tabla 2 muestra el porcentaje de universidades que desarrollan alguna de las actividades estudiadas. La tabla 3 recoge el porcentaje 
Tabla 2

Porcentaje de universidades por número de actividades que desarrollan $(N=34)$

\begin{tabular}{ll}
\hline Universidades que desarrollan & \\
Una actividad & 19,6 \\
Dos actividades & 23,9 \\
Tres actividades & 6,5 \\
Cuatro actividades & 13 \\
Cinco actividades & 2,2 \\
\hline
\end{tabular}

Fuente: Elaboración propia.

Tabla 3

Porcentaje de universidades que desarrollan cada actividad $(N=34)$

\begin{tabular}{ll}
\hline Universidades que desarrollan & \\
Cursos cero & 53 \\
Plataformas web de aprendizaje & 15,2 \\
Planes de acción tutorial de matemáticas & 37,5 \\
Grupos de trabajo & 40 \\
Formación del profesorado & 10,9 \\
Colaboración con sociedades de profesores & Sin datos globales \\
Actividades con colectivos específicos & 10,9 \\
\hline
\end{tabular}

Fuente: Elaboración propia.

de universidades que desarrollan las distintas actividades estudiadas.

A continuación, se realiza una descripción más pormenorizada de las actividades estudiadas y sus características más peculiares. En la web del proyecto (http://www.unioviedo.es/vee) se pueden encontrar los detalles pormenorizados de cada una de las actividades en cada universidad.

\section{Cursos cero}

Son la actividad que más se desarrolla en las universidades estudiadas. Realizando un recuento global, un 53\% de las universidades estudiadas posee un curso cero. Dentro de este porcentaje, solo un $24 \%$ de los cursos cero ha sido transformado en una asignatura dentro del plan de grado al adaptar el currículo al Espacio Europeo, siendo mayoritario el modelo de curso previo, de clases expositivas y resolución de ejercicios y realizado con anterioridad al curso lectivo. Atendiendo al tipo de docencia realizada, un $12 \%$ de los cursos cero se realizan de manera virtual, frente al mayoritario $89 \%$, que mantiene el modelo presencial. Se encuentran casos de transformación en cursos virtuales del curso tradicional (Universidad Politécnica de Madrid), de transformación del curso tradicional en una asignatura obligatoria del plan de estudios (Universidad Complutense) o en asignatura optativa (Barcelona).

Atendiendo a la organización de los cursos, en todos los casos le corresponde a una determinada facultad o, si se realizan de manera transversal para varias titulaciones, al vicerrectorado competente en materia de estudiantes. Respecto a las fuentes de financiación de los cursos, estas son muy variadas y, además, como se señaló anteriormente, los datos proporcionados por las universidades son muy imprecisos (se entiende que no hay una voluntad de opacidad, sino que realmente no están contabilizados analíticamente). De manera exacta, se puede determinar el origen de la financiación en los cursos que han sido transformados en asignaturas, pues tienen un coste por crédito asignado vinculado a la matrícula del alumnado. En otros casos la financiación puede proceder de diversas fuentes: en el $88 \%$ de los casos, se trata de financiación a cargo de los presupuestos de los departamentos o centros que los organiza. Esta fuente se puede complementar con otra, que en ocasiones actúa de manera exclusiva: el 16\% de los cursos reciben financiación con cargo a los presupuestos de algún vicerrectorado. Durante el período estudiado también se detectaron algunos casos en los cuales los cursos se financiaron a través de convenios con el Ministerio de Educación para el desarrollo de planes de acción tutorial.
Si se presta atención a los costes, en su mayor parte se trata de costes derivados de compensar las horas de docencia extra que realiza el profesorado, o bien (mucho más difícil de contabilizar) de computar estas horas dentro de la dedicación docente.

En cuanto a la evaluación, se ha encontrado que en un $68 \%$ de los cursos no hay evaluación del profesorado y, en el $20 \%$ que sí la hay, consiste únicamente en una encuesta al alumnado sobre el desempeño de los docentes (el 12\% restante no respondió a este apartado). Se considera importante hacer notar que no se ha constatado en ningún caso que el profesorado universitario haya recibido formación específica relacionada con los cursos cero. Por otro lado, un $40 \%$ de los cursos no evalúa a los estudiantes al finalizar la docencia, frente a un $44 \%$ que sí; los instrumentos de evaluación detectados han sido exámenes, ejercicios entregables o test de autoevaluación. En un 64\% de los casos el alumnado no realiza ningún tipo de evaluación sobre el desarrollo del curso; en los casos restantes, esta consiste en una encuesta de satisfacción (en la que se suele evaluar el desempeño del profesorado).

\section{Plataformas web de aprendizaje}

Están siendo una alternativa para los antiguos cursos cero. De hecho, 2 universidades han transformado su curso cero en una plataforma web de aprendizaje con material y ejercicios disponibles a lo largo de todo el curso escolar (Granada y Oviedo).

Actualmente, un 15,2\% de las universidades declara poseer una plataforma de estas características. En todos los casos, las plataformas están sostenidas con fondos propios y son mantenidas por profesorado voluntario.

\section{Planes de acción tutorial}

En cuanto a los planes de acción tutorial (PAT), se ha encontrado que el $37,5 \%$ de las universidades posee uno directamente relacionado con matemáticas. Su funcionamiento es muy diverso, encontrándose PAT donde el tutor es un docente o un alumno de último curso de grado. Atendiendo a los tutorados, en un 33\% de los casos se trata de estudiantes de nuevo ingreso, frente al 67\% restante donde se desarrolla el PAT durante todo el grado. Es variable también el número de estudiantes por tutor, encontrándose una horquilla que va desde los 4 a los 30 alumnos por tutor. Esta ratio no depende de que el tutor o mentor sea un profesor o un estudiante.

Siete universidades $(38,8 \%$ del total) indican que cuentan con cursos o jornadas de formación para los tutores que participan en el PAT. En un 33,3\% de los casos existe una evaluación del PAT, bien a través del informe de calidad de grado (Girona), a través de encuestas de satisfacción al alumnado (Barcelona o Santiago) o también al profesorado (Cádiz).

La financiación que reciben los PAT es muy diversa y difícilmente cuantificable. Señalamos como dato importante que en un $72 \%$ de los casos los encuestados afirman que cuentan con financiación, mientras que un $22,2 \%$ manifiesta que se financia a través de los recursos propios de la facultad o escuela.

Respecto a la existencia de algún tipo de reconocimiento para los tutores, se ha encontrado que un $28 \%$ de las universidades reconocen de diversas maneras la actuación de los tutores (reconocimiento económico o docente).

\section{Grupos de trabajo}

Se ha constatado la existencia grupos de trabajo en un $40 \%$ de las universidades. Se distinguen entre ellos los dirigidos a estudiantes universitarios (31,6\%) y a los preuniversitarios (36,8\%), con 2 universidades que tienen grupos de ambos niveles (11\%), y también con ausencia de respuesta a esta pregunta (20,6\%). Los dirigidos a estudiantes universitarios realizan, por lo general, tareas 
de entrenamiento matemático, refuerzo de matemáticas u orientación académica. Los dirigidos a los estudiantes preuniversitarios son mucho más variados, con tareas como estimulación del talento matemático, campus matemáticos de verano, semana científica o grupos de resolución de problemas.

\section{Formación del profesorado de matemáticas}

La formación de profesorado universitario sobre secundaria suele ser de carácter voluntario y canalizada a través de cursos ofertados por los Institutos de Ciencias de la Educación. Se ha constatado la existencia en 5 universidades de un plan de formación sobre el conocimiento mutuo de las etapas de secundaria y universidad. En todos los casos se trabaja de forma conjunta, a través de jornadas, cursos y puntos de encuentro, con el fin de intercambiar información sobre los 2 niveles. La Universidad de Murcia, por ejemplo, cuenta con un grupo de trabajo cuyo objetivo es el estudio de los problemas de coordinación entre ambas etapas.

\section{Trabajo con sociedades profesionales}

El profesorado universitario colabora, normalmente de manera voluntaria, en y con sociedades de profesores de matemáticas. Este carácter voluntario genera que se encuentre una enorme variedad de actuaciones: organización de conferencias, talleres, jornadas, programas de estimulación del talento matemático (por ejemplo, ESTALMAT), cursos de formación a profesorado de secundaria, organización de la olimpiada matemática.

La dispersión y variedad de las actividades, así como el carácter netamente individual de las colaboraciones llevan a no considerar de manera global los datos de participación en la tabla 3.

\section{Colectivos específicos}

Finalmente, se estudió el trabajo con colectivos específicos, como es el alumnado procedente de formación profesional y los estudiantes que acceden por vías como mayores de 25, o 45 años, o por mayores de 40 años con experiencia laboral. Estos colectivos suelen presentar dificultades, a veces graves, en su incorporación a estudios de grado, principalmente en escuelas de ingeniería, donde se suelen concentrar suspensos en las asignaturas básicas. La única actividad específica dedicada a este colectivo son los cursos de preparación de la prueba de acceso para mayores de 25 años y solo se realiza en 5 universidades.

\section{Competencia matemática}

La importancia de la programación por competencias y resultados de aprendizaje se deriva de su obligatoriedad, establecida por el Real Decreto 1393/2007, de 29 de octubre, por el que se establece la ordenación de las enseñanzas universitarias oficiales.

Sin embargo, la información recogida muestra que el número de universidades que enfocan su metodología o planificación docente en la adquisición de competencias es realmente muy pequeño. La tabla 4 resume los datos.

Cuando se utiliza una programación por competencias, se definen a partir de las expresadas en la Ley Orgánica de Educación (LOE) o en el primer curso del grado (por ejemplo, en la Universidad Autónoma de Barcelona), o las definen los grupos de trabajo (Universidades de Castilla-La Mancha, Granada o Lleida).

\section{Discusión}

Antes de entrar en la discusión de los resultados, es oportuno subrayar la alta tasa de respuesta en las universidades públicas españolas (el 74\%). Se trata de un dato altamente satisfactorio, y muy superior a la participación media en estudios similares (Ayuga et al., 2008; Barrenetxea et al., 2008), que refuerza la extrapolación de las conclusiones al conjunto de las 46 universidades públicas consideradas.

Es muy importante comenzar la discusión reseñando que el propio proceso de recopilación de los datos ha puesto de manifiesto debilidades del sistema universitario, principalmente la disgregación en organización y estructura de la información interna. Hay actividades desarrolladas en una facultad o escuela sin colaborar con otros servicios universitarios, otras son desarrolladas por servicios centrales sin conocimiento de las facultades, hay incluso actividades desarrolladas a título individual por profesorado de un departamento. Esta disgregación de los datos no solo dificulta su recogida y posterior análisis, sino que evidencia que dentro de las universidades se realizan actividades paralelas, complementarias e incluso contrapuestas dirigidas a fines que son similares, sin que exista un control o una coordinación general.

La segmentación de la información tiene varias consecuencias indeseadas: deficiencia en la formación específica que reciben los docentes, ausencia de evaluación de las actividades, no medición de la efectividad y, en general, desconocimiento del impacto en el rendimiento del alumnado que participa. Y por ello, supone una importante limitación al alcance de la investigación que se planteaba, cuyo objetivo era identificar y evaluar las acciones de las universidades españolas para mejorar el rendimiento en las asignaturas de matemáticas de los nuevos estudiantes.

Por lo general, no hay una evaluación de las actividades ni del personal involucrado en ellas, más allá de un cuestionario de satisfacción por parte del alumnado (y, ocasionalmente, de la satisfacción del profesorado). Esto explica, en parte, la falta de estudios sistemáticos sobre la efectividad de estas actividades.

Por otro lado, plantear un estudio sobre la efectividad es realmente complejo. Resulta difícil diseñar la actuación para poder contar con un grupo de control que permita un mínimo análisis estadístico. Al tratarse de actividades que se ofrecen a todo el alumnado, es problemático impedir el acceso de unos alumnos y favorecer el de otros. Cuando la participación del alumnado es voluntaria, no se puede asumir que haya idénticas condiciones de partida entre el alumnado que participa y el que no, por lo que los estudios sobre la eficacia de la actuación quedan limitados.

En el caso concreto de las plataformas web de aprendizaje es difícil cuantificar la relación entre el esfuerzo o la dedicación invertida en la plataforma y las notas finales del alumnado. Sin embargo, sí se puede determinar fácilmente el número de accesos a las webs, la participación por actividad propuesta y el tiempo invertido para realizarlas. En estos casos, se constata que la motivación por parte del profesorado es fundamental para conseguir que el alumnado se involucre más con la actividad.

Cuando los cursos cero han sido transformados en asignaturas obligatorias, sí se ha evidenciado una mejoría en la preparación del alumnado para afrontar el resto de materias del grado, basada en el mejor rendimiento académico. En cursos cero tradicionales, intensivos y voluntarios, más allá de comprobar las notas en las asignaturas de primer curso de los estudiantes que lo han seguido, no se han establecido otras medidas comparativas respecto a los estudiantes que no lo han seguido o respecto a la correlación que pueda existir entre la nota inicial del alumno y su rendimiento tras el curso cero. Es decir, no hay constatación de que los estudiantes que siguen el curso cero rindan mejor por el hecho de haber realizado este curso.

Por su lado, los grupos de trabajo con preuniversitarios están dirigidos a un colectivo de alumnos y alumnas muy específico: altas capacidades matemáticas o un gran interés y motivación por ellas. Esto induce un sesgo de partida en los participantes, que produce que las actuaciones llevadas a cabo no contribuyan a mejorar la competencia matemática de los estudiantes con un 
Tabla 4

Porcentaje de universidades que desarrollan programación por competencias según actividad $(N=34)$

\begin{tabular}{|c|c|c|c|c|c|c|}
\hline ¿Programación por competencias? & Cursos cero & Grupos de trabajo & Plataformas web & Planes tutoriales & Colectivos específicos & Formación del profesorado \\
\hline Sí & 11,1 & 42,1 & 42,86 & 22,22 & 0 & 0 \\
\hline No & 88,8 & 10,53 & 42,86 & 33,33 & 100 & 100 \\
\hline No se sabe & 0 & 47,37 & 14,29 & 44,45 & 0 & 0 \\
\hline
\end{tabular}

Fuente: Elaboración propia.

comportamiento medio o con un bajo rendimiento matemático, quienes no suelen participar en estas actividades. En cuanto a los grupos de trabajo con universitarios, se observa que se centran más en reforzar los conocimientos o técnicas matemáticas del alumnado de nuevo ingreso que pueda presentar dificultades o problemas en el aprendizaje. Es decir, se apoya a los estudiantes aventajados antes de llegar a la universidad, pero una vez en ella, apenas se les presta atención. Finalmente, en los PAT se constata una nula definición de las competencias requeridas por el profesor-tutor o el estudiantementor (como, por ejemplo, la que desarrolla Vélaz de Medrano, 2008, para orientadores).

La valoración económica de estas actividades ha sido también muy difícil de cuantificar, debido a la falta de registros por parte de las universidades, por lo que la información obtenida puede estar incompleta. Ha de señalarse al respecto la aún escasísima implantación que la contabilidad analítica tiene en el sistema universitario (especialmente el público), por lo que resulta imposible determinar exactamente el coste de la actividad.

En cuanto a la financiación, se ha comprobado que proviene principalmente de la universidad, computándose en los presupuestos de una facultad, un departamento o un vicerrectorado. Algunas actividades, como la tercera parte de los cursos cero estudiados, conllevan una matrícula aunque no sean asignaturas oficiales, por lo que se añade así una fuente extra de financiación de los costes.

Sobre la base del análisis de los datos recogidos, se han seleccionado una serie de actuaciones de entre todas las examinadas, que se considera que pueden constituir un compendio de buenas prácticas.

\section{Adaptación de los cursos cero a los grados}

Durante los años en los que el bachillerato LOGSE estuvo implantado en convivencia con los estudios previos al Espacio Europeo surgieron los denominados cursos cero o de nivelación, desarrollados de manera intensiva y previa al comienzo del curso académico, como un apoyo al estudiantado que carecía de los requisitos necesarios para cursar las asignaturas matemáticas del primer curso universitario.

Sin embargo, con la implantación casi simultánea del bachillerato LOE y de los estudios de grado, algunas universidades han adaptado las asignaturas de matemáticas en los primeros cursos de grado para ajustarlas al currículo de bachillerato, contribuyendo a la reducción del fracaso de los estudiantes de nuevo ingreso en las asignaturas de matemáticas. En otros casos, como en la asignatura de Matemáticas Básicas del Grado en Matemáticas de la Universidad Complutense de Madrid, se ha introducido en el currículo de grado una asignatura preparatoria, obligatoria, intensiva y previa al resto de materias de primer curso, con la finalidad de dotar al alumnado del conocimiento y de las herramientas matemáticas necesarios para afrontar las otras materias.

En aquellos casos en los que no se haya abordado esta sincronización, se considera imprescindible que debe realizarse, ya que no se puede exigir un conocimiento previo a quien no ha tenido oportunidad de adquirirlo. Dicho de otro modo, si esos contenidos son necesarios, deberían abordarse dentro del grado como una formación común para todos los estudiantes.
Además del ajuste de contenidos, se está produciendo en las universidades una adaptación metodológica. En general, está siendo más lenta de lo inicialmente previsto, en muchos casos por la falta de apoyo institucional y de incentivos al reciclaje docente del profesorado, y por las dificultades económicas para formar grupos de tamaño reducido. A pesar de la lentitud del avance, es indiscutible que el profesorado universitario en general, y particularmente el de matemáticas, está adaptando las metodologías docentes para seguir aquellas que más favorecen el aprendizaje basado en competencias. Este hecho debería contribuir también a favorecer la incorporación de estos cursos cero como parte de los estudios de grado.

En resumen, partiendo de la línea expuesta por Clark y Lovric (2001, p. 33): "transition programme, thus, must incorporate a process of 'unlearning', i.e., abandoning, or at least weakening cognitive models inhabited by misconceptions» se considera que estos períodos de desaprendizaje y adaptación a nuevas circunstancias se amoldan mejor a una secuenciación del aprendizaje dentro del propio grado universitario.

\section{Coordinación secundaria-universidad}

Se ha observado que la colaboración con secundaria desde la universidad depende de la voluntad del profesorado para colaborar y de un interés a título personal. Sin embargo, a menudo se señala como fundamental esta coordinación (recientemente, en Lorenzo Moledo, Argos González, Hernández García y Vera Vila, 2014).

Los autores consideran que se debe fomentar la creación de grupos de trabajo estables entre profesorado de ambos niveles, reconociéndolos de manera oficial como grupos de innovación educativa o docente. En este sentido, la Universidad de Oviedo lleva varios años apostando por "Proyectos puente» de innovación educativa sobre el paso del Bachillerato a la Universidad, incluyendo uno de matemáticas (algunos de cuyos resultados se recogen en Huidobro, Méndez y Serrano, 2010).

Por otro lado, la Universidad debería promover actividades de formación de profesorado en temas relacionados con secundaria y el paso a la universidad, pues los cuestionarios reflejan la carencia de formación de profesorado en lo que se aleja de su práctica docente habitual.

\section{Plataformas web de aprendizaje}

Se están revelando como una herramienta de gran utilidad, pues permiten el aprendizaje sostenido a lo largo del curso escolar, adaptándose a las necesidades del alumnado en el momento en el que estas se producen. Están diseñadas como una plataforma de refuerzo, como un complemento a la adaptación, no como apoyo a la docencia reglada. En ellas, el material de consulta, al contrario que en los cursos cero, está disponible a lo largo de todo el curso escolar, por lo que el alumnado puede consultarlo en cualquier momento y realizar las tareas de autoevaluación, refuerzo o ampliación propuestas. En este sentido, de cara a la adquisición de competencias, las tareas se pueden orientar con metodologías adecuadas que, al no tener el límite temporal de los cursos cero, pueden contribuir más favorablemente a su adquisición (trabajo colaborativo o aprendizaje autónomo). 
Algunas plataformas de matemáticas (Universidad Politécnica de Madrid) cuentan con un largo recorrido interno y ya se han abierto al público en general, mientras que otras (Universidad de Oviedo) aún están siendo ofrecidas únicamente para su alumnado, aunque en ambos casos el apoyo institucional para su éxito es fundamental.

\section{Estabilidad del profesorado}

El trabajo con un profesorado estable que participe de manera regular en las actividades mejoraría sustancialmente el funcionamiento, pues conocería las debilidades de la actividad y ayudaría a crear un sistema de retroalimentación que aumentaría la efectividad. Whannell y Allen, 2012 recientemente han demostrado la importancia que la actitud y la forma de actuar del profesorado tiene en el éxito de su alumnado en matemáticas en el primer año de la universidad. Ello refuerza nuestra apuesta por una formación adecuada del profesorado en el aprendizaje por competencias (véase Ion y Cano, 2012) y por lograr una plantilla estable especializada en esta transición mediante formación en las especificidades del currículo matemático en secundaria.

A continuación se sugieren unas propuestas para la mejora de la transición matemática de la secundaria a la universidad, basadas en el estudio realizado y que pueden ser utilizadas como líneas de trabajo futuro en la evaluación de las actividades.

\section{Identificación de las dificultades del alumnado}

La ausencia de estudios sobre la efectividad de las actividades enmascara el hecho de que pueden no ser suficientes o no estar funcionando correctamente. Por ello, el paso previo imprescindible al diseño de actuaciones debe ser el estudio detallado de cuáles son las dificultades del alumnado o si existe algún tipo de carencia formativa asociada a algún colectivo específico (Jiménez y Márquez, 2014, analizan el problema de los estudiantes mayores desde una perspectiva global, pero no hay estudios sobre dificultades matemáticas). Para ello deben diseñarse, en colaboración con expertos en investigación educativa y didáctica, cuestionarios que sean capaces de identificar el tipo de problema que afecta al estudiante.

La introducción de pruebas finales de evaluación en cada etapa educativa en el marco de la Ley Orgánica de Mejora de la Calidad en la Enseñanza (LOMCE) puede ayudar a detectar estas dificultades con carácter general y a nivel nacional. La opinión de los formadores tanto en el bachillerato como en la universidad debe también ser tenida en cuenta en la identificación de estos problemas. En todo caso, sin una adecuada evaluación resulta imposible definir adecuadamente la intervención necesaria para corregir los problemas.

\section{Uso compartido de plataformas web}

Teniendo en cuenta la concurrencia de objetivos y de metodologías, deberían aunarse esfuerzos para propiciar una plataforma web conjunta o el uso compartido de las actuales plataformas de matemáticas. De este modo, y teniendo en cuenta proyectos similares para otras etapas educativas (como el Proyecto Descartes), se podría crear una plataforma que multiplicase su alcance y, al mismo tiempo, redujese los costes de gestión, como las existentes para los campus virtuales compartidos (Cabrero y Marín, 2011).

\section{Registros sobre el coste de las actividades}

A medida que en las universidades se vaya implantando la contabilidad analítica, debe realizarse un registro efectivo del coste de las actividades realizadas. A pesar de las dificultades, este tipo de registro es crucial para poder valorar la efectividad de las actuaciones llevadas a cabo.

\section{Reconocimiento al profesorado}

Todas las actividades que se han estudiado evidencian un mejor funcionamiento cuando la actividad del profesorado se reconoce por alguna vía. No solo se tienen en cuenta medidas económicas, sino reconocimiento en la dedicación docente o en la carrera profesional.

\section{Medidas de eficacia}

Es necesario definir indicadores que puedan permitir valorar la eficiencia de las actuaciones llevadas a cabo. Más allá de las serias dificultades que se han puesto de manifiesto en este artículo, resulta imperativo trabajar en esta línea para poder afianzar medidas de eficiencia del esfuerzo que las instituciones están realizando.

A pesar del alto número de universidades que están llevando a cabo actuaciones para mejorar la competencia matemática de los estudiantes de nuevo ingreso, se observa una gran dispersión en cuanto a coordinación y dependencia orgánica en ellas. Esta falta de coordinación conlleva una gran dificultad a la hora de determinar medidas generales como la relación coste-eficiencia y la influencia en el rendimiento del alumnado.

Aun así, se ha realizado un reconocible esfuerzo por adaptar los grados a la formación matemática previa del alumnado, concretándose en algunos ejemplos de asignatura introductoria, con carácter obligatorio e incardinada en el plan de estudios.

La actividad on-line ha crecido enormemente, bien a través del soporte de los campus virtuales, bien gracias a las plataformas web de aprendizaje, concebidas como soporte del aprendizaje del alumnado con dificultades en su primer contacto con la matemática a nivel universitario. Se centran principalmente en el refuerzo de contenidos de bachillerato que el alumnado debería manejar con soltura y proporcionan, asimismo, materiales de autoevaluación.

El trabajo conjunto entre profesorado de secundaria y universidad se revela como una pieza muy importante en la motivación del alumnado, bien en grupos formados por profesores o bien guiando grupos de trabajo de alumnado.

Los PAT son otra herramienta que comienza a ser utilizada no solo desde el punto de vista general sino específicamente para detectar problemas relacionados con las matemáticas y para adquirir competencias vinculadas al trabajo colaborativo en la resolución de problemas.

Además de lo señalado en el catálogo de buenas prácticas, resulta interesante subrayar la necesidad de un trabajo previo de detección e identificación de las dificultades concretas del alumnado, estudiando posibles asociaciones de los problemas localizados con perfiles de alumnado, que permitan dar una respuesta más personalizada que la actual y más dirigida a colectivos específicos como los estudiantes procedentes de formación profesional y mayores de 25/40/45 años.

Por último, ha de subrayarse el importante esfuerzo para el desarrollo de webs de aprendizaje, no solo económico sino humano, por la enorme cantidad de horas de trabajo que el profesorado dedica y que habitualmente se realiza de manera altruista. Este esfuerzo aumentaría su efecto si, como se ha señalado anteriormente, se promueven plataformas conjuntas.

Como se manifestó anteriormente, la principal limitación del estudio es, a juicio de los autores, la dificultad para recopilar de manera centralizada la información dentro de cada universidad, producida por la enorme dispersión organizativa de las actividades y de los datos derivados de ellas. Por otro lado, de cara a futuros trabajos en la línea que se ha iniciado en el presente artículo, sería interesante la definición de las medidas de eficacia que se 
mencionan para analizar más pormenorizadamente las actividades una a una. De modo más concreto, de cara a la evaluación de los cursos cero resulta imprescindible el seguimiento del alumnado que realiza los cursos y el estudio de su situación de partida.

\section{Financiación}

Proyecto EA2011-0108, de Estudios y Análisis, del Ministerio de Educación, Cultura y Deporte.

\section{Conflicto de intereses}

Los autores declaran no tener ningún conflicto de intereses.

\section{Agradecimientos}

Los autores agradecen a Lluis Miquel García-Raffi, Raquel Mallavibarrena, Roberto Muñoz y Elena Thibaut su aportaciones a la discusión. Asimismo, agradecen a los revisores de la revista sus sugerencias de mejora.

\section{Referencias bibliográficas}

Anthony, G. (2000). Factors influencing first year students' success in mathematics. International Journal of Mathematical Education in Science and Technology, 31(1), 3-14.

Artigue, M. (2001). What can we learn from research at the university level. In D. Holton (Ed.), The teaching and learning of mathematics at the university level: An ICMI study (237-254). Dordrecht. The Netherlands: Kluwer Academic Publishers.

Ayuga, E., González, G., Grande, M. A., Martín, S., Moral, J. M., Tévar, G., et al. (2008). Instrumentos utilizados por las universidades públicas españolas para facilitar la transición de la Enseñanza Secundaria a la Universidad. Madrid: Ministerio de Educación, Cultura y Deporte. Recuperado de http://138.4.83.162/mec/ayudas/CasaVer.asp?P=29 318

Barrenetxea, M., Barandiaran, M., Cardona, A., Martín, A. M., Mijangos, J. J. y Olaskoaga, J. (2008). Las políticas informativas orientadas a estudiantes de enseñanzas preuniversitarias en las universidades españolas. Madrid: Ministerio de Educación, Cultura y Deporte. Recuperado de http://138.4.83.162/ mec/ayudas/CasaVer.asp?P=29 357.

Barnard, D. (2003). The transition to mathematics at university: Students' views. New Zeland Journal of Mathematics, 32(1-8).

Cabrero, J. y Marín, V. (2011). Campus virtuales compartidos (CVC): Análisis de una experiencia. Educación XX1, 14(2), 111-132.

Clark, M. y Lovric, M. (2008). Suggestion for a theorical model for secondarytertiary transition in mathematics. Mathematics Education Research Journal, 20, 25-37.
De Guzmán, M., Hodgson, B. R., Robert, A. y Villani, V.(1998). Difficulties in the passage from Secondary to Tertiary Education. Proceedings of the ICM, Berlin (Extra Volume ICM III).

Díaz, P. y Rodríguez-Muñiz, L. J. (2013). Experiences in Spanish universities to reduce failure in Mathematics of incoming students. Procedia - Social and Behavioral Sciences, 93, 2006-2009.

Hourigan, M. y O'Donoghue, J. (2007). Mathematical under-preparedness: The influence of the pre-tertiary mathematics experience on students' ability to make a successful transition to tertiary mathematics courses in Ireland. International Journal of Mathematical Education in Science and Technology, 38(4), 461-476.

Huidobro, J. A., Méndez, M. A. y Serrano, M. L. (2010). Del Bachillerato a la Universidad: las matemáticas en las carreras de ciencias y tecnología. Aula Abierta, 38(1) 71-80.

Ion, G. y Cano, E. (2012). La formación del profesorado universitario para la implementación de la evaluación por competencias. Educación XX1, 15(2), 249-270.

(2008). IV Escuela de Educación Matemática Miguel de Guzmán (Madrid 21-24 de julio de 2008). Documento de conclusiones. Madrid: Universidad Rey Juan Carlos [consultado 12 Jul 2012]. Disponible en: http://www.escet.urjc.es/ satellite/iv\%20escuela\%20miguel\%20de\%20guzman.html

Jiménez Rodrigo, M. L. y Márquez Lepe, E. (2014). Ir a la Universidad después de los 30: dificultades y factores facilitadores. Aula Abierta, 42(1), 1-8.

Kajander, A. y Lovric, M. (2005). Transition from secondary to tertiary mathematics: McMaster University experience. International Journal of Mathematics Education in Science and Technology, 36(2-3), 149-160.

Lorenzo Moledo, M., Argos González, J., Hernández García, J. y Vera Vila, J. (2014). El acceso y la entrada del estudiante a la universidad: Situación y propuestas de mejora facilitadoras del tránsito. Educación XX1, 17(1), 15-38.

McInnes, C., James, R. y Hartley, R. (2000). Trends in the first year experience in Australian universities. Canberra: AGDS.

Sánchez, M. F., Guillamón, J. R., Ferrer-Sama, P., Martín, A. M., Pérez, J. C. y Villalba, E. (2008). Situación actual de los servicios de orientación universitaria: estudio descriptivo. Revista de Educación, 345, 329-352.

Selden, A. (2005). New developements and trends in tertiary mathematics education: Or more of the same? International Journal of Mathematical Education in Science and Technology, 36(2-3), 131-147.

Taylor, J. A. (2008). Assessment in first year university: A model to manage transition. Journal of University Teaching \& Learning Practice,, 5(1)

Velasco, A. y Benito, A. (2011). La mentoría entre iguales en la Universidad Europea de Madrid: una estrategia educativa para el desarrollo de 35 competencias generales y específicas. Higher Learning Research Communications, 1(1), 10-32.

Vélaz de Medrano, C. (2008). Competencias del profesor-mentor para el acompañamiento al profesorado principiante. Profesorado. Revista de curriculum y formación del profesorado, 13(1), 210-229.

Vieira, M. J. y Vidal, J. (2006). Tendencias para la educación superior europea e implicaciones para la orientación universitaria. Revista Española de Orientación y Psicopedagogía, 17(1), 75-97.

Whannell, R. y Allen, B. (2012). First year mathematics at a regional university: Does it cater to student diversity? The International Journal of the First Year in Higher Education, 3(2), 45-85.

Wood, L. (2001). The Secondary-Tertiary interface. In D. Holton (Ed.), The teaching and learning of mathematics at University level: An ICMI study (87-98). Dordrecht The Netherlands: Kluwer Academics Publishers. 